Skin Appendage Disord 2020;6:175-176

DOI: $10.1159 / 000505820$

\section{Low-Dose Oral Minoxidil for Female Pattern Hair Loss: A Unicenter Descriptive Study of 148 Women}

\author{
Rita Rodrigues-Barata $a^{\mathrm{a}, \mathrm{b}}$ Oscar M. Moreno-Arrones ${ }^{\mathrm{a}, \mathrm{b}}$ \\ David Saceda-Corralo ${ }^{\mathrm{a}, \mathrm{b}}$ Juan Jiménez-Cauhéa \\ Daniel Ortega-Quijano a Diego Fernández-Nieto ${ }^{\text {a }}$ \\ Pedro Jaén-Olasolo ${ }^{\mathrm{a}, \mathrm{b}}$ Sergio Vaño-Galvan ${ }^{\mathrm{a}, \mathrm{b}}$ \\ aTrichology Unit, Dermatology Department, Ramon y Cajal \\ University Hospital, Madrid, Spain; ${ }^{b}$ Trichology Unit, Grupo de \\ Dermatología Pedro Jaen, Madrid, Spain
}

\section{Dear Editor,}

Female pattern hair loss (FPHL) affects more than $50 \%$ of women by the age of 80 years [1] and may produce a negative impact on quality of life [2]. Treatment is challenging, as topical minoxidil is the only approved therapy by the Food and Drug Administration and European Medicine Agency [3]. Although this treatment is effective, its long-term compliance is suboptimal because it may produce contact dermatitis, altered hair texture, and difficulty with hair styling. The usefulness of low-dose oral minoxidil (LDOM) for FPHL has been previously described in few reports [4-6]. The objective of our study was to describe the effectiveness and safety of LDOM in women with FPHL in clinical daily practice.

A retrospective study was designed with patients diagnosed clinically and by trichoscopy with FPHL who were receiving LDOM for a minimum of 6 months as monotherapy or associated with other treatments. Of the patients that were also receiving other therapies, only those without therapeutic or clinical changes in the last 12 months were included. Clinical response was evaluated by 3 independent dermatologists comparing global digital photographs before and after treatment using a 4-point ordinal scale (worsening, stabilization, mild improvement, and marked improvement). A marked improvement was defined as the improvement in two grades or more on the 5-point Sinclair scale. The complete observer's agreement was necessary to classify a patient as "improvement."

A total of 148 FPHL women with a mean age of 47.2 years (range 17-85) receiving LDOM were included. Doses between 0.25 and $2 \mathrm{mg}$ of minoxidil were used daily (median $1 \mathrm{mg}$ daily), for a mean time of 9 months (range 6-27). A total of 23 patients (15.5\%) received LDOM as monotherapy, while 125 patients $(84.5 \%)$ received other concomitant therapies (Table 1). Regarding effectiveness, 30 patients $(20.3 \%)$ presented stabilization of their alopecia and 118 patients $(79.7 \%)$ presented clinical improvement. Of these, 95 patients $(64.2 \%)$ presented a slight improvement and 23
Table 1. Baseline characteristics of the cohort of 148 women with FPHL treated with LDOM

\begin{tabular}{|c|c|c|}
\hline Mean age and range & 47.2 years $(17-85)$ & \\
\hline \multirow{4}{*}{$\begin{array}{l}\text { Severity of } \\
\text { alopecia }\end{array}$} & Sinclair II & 100 patients $(67.6 \%)$ \\
\hline & Sinclair III & 22 patients $(14.9 \%)$ \\
\hline & Sinclair IV & 16 patients $(10.8 \%)$ \\
\hline & Sinclair V & 10 patients $(6.7 \%)$ \\
\hline \multirow{4}{*}{$\begin{array}{l}\text { Dose of oral } \\
\text { minoxidil }\end{array}$} & $0.25 \mathrm{mg}$ daily & 3 patients $(2 \%)$ \\
\hline & 0.5 mg daily & 60 patients $(40.5 \%)$ \\
\hline & $1 \mathrm{mg}$ daily & 84 patients $(56.8 \%)$ \\
\hline & 2 mg daily & 1 patient $(0.7 \%)$ \\
\hline \multirow{10}{*}{$\begin{array}{l}\text { Other } \\
\text { concomitant therapies } \\
\text { ( } n=125 \text { patients) }\end{array}$} & Oral dutasteride & 89 patients $(71.2 \%)$ \\
\hline & $\begin{array}{l}\text { Mesotherapy with } \\
\text { dutasteride }\end{array}$ & 27 patients $(21.6 \%)$ \\
\hline & Topical 5\% minoxidil & 18 patients $(14.4 \%)$ \\
\hline & Platelet-rich plasma & 15 patients $(12 \%)$ \\
\hline & Oral finasteride & 5 patients $(4 \%)$ \\
\hline & Oral flutamide & 4 patients $(3.2 \%)$ \\
\hline & Oral bicalutamide & 2 patients $(1.6 \%)$ \\
\hline & Oral cyproterone acetate & 2 patients $(1.6 \%)$ \\
\hline & Low-level light therapy & 1 patient $(0.8 \%)$ \\
\hline & Topical latanoprost & 1 patient $(0.8 \%)$ \\
\hline
\end{tabular}

patients $(15.5 \%)$ a marked improvement. The clinical improvement was higher in more advanced stages of alopecia $(p=0.026)$. Of the subgroup of patients receiving LDOM in monotherapy $(n=23), 12(52 \%)$ presented a slight improvement and $3(13 \%)$ a marked improvement (Fig. 1). No patients worsened. Adverse effects were observed in 29 patients (19\%), mainly hypertrichosis (25 patients, 17\%). Other adverse effects included tachycardia (2 patients) and lower limb edema and general malaise in 1 patient. Only these 3 patients (2\%) withdrew from treatment. On multivariate analysis, we could not demonstrate a statistically significant difference in the effectiveness or safety of LDOM by dosage or patients' age.

In the present study, we described the largest series of patients with FPHL treated with LDOM to date. In a previous report about LDOM for FPHL, similar results in terms of efficacy and tolerance were observed combining $0.25 \mathrm{mg}$ of LDOM with $25 \mathrm{mg}$ of spironolactone [4]. Our results support that LDOM may be an effective treatment in FPHL, with a higher degree of response in more advanced stages, and a good safety profile. One of the major advantages of the oral intake is the improvement of long-term compliance. The main limitation of our study is the concomitant use of other therapies in a subgroup of patients. However, it is worth karger@karger.com

www.karger.com/sad

(C) 2020 S. Karger AG, Base

Karger ${ }^{\prime \prime}=$
Rita Rodrigues-Barata

Dermatology Department, Ramon y Cajal University Hospital

Carretera Colmenar Viejo km 9.100

ES-28034 Madrid (Spain)

rita.rodrigues.md@gmail.com 
Fig. 1. a Female pattern hair loss grade IV in a 28 -year-old woman. b Great improvement after 6 months of treatment with LDOM $1 \mathrm{mg}$ daily in monotherapy.
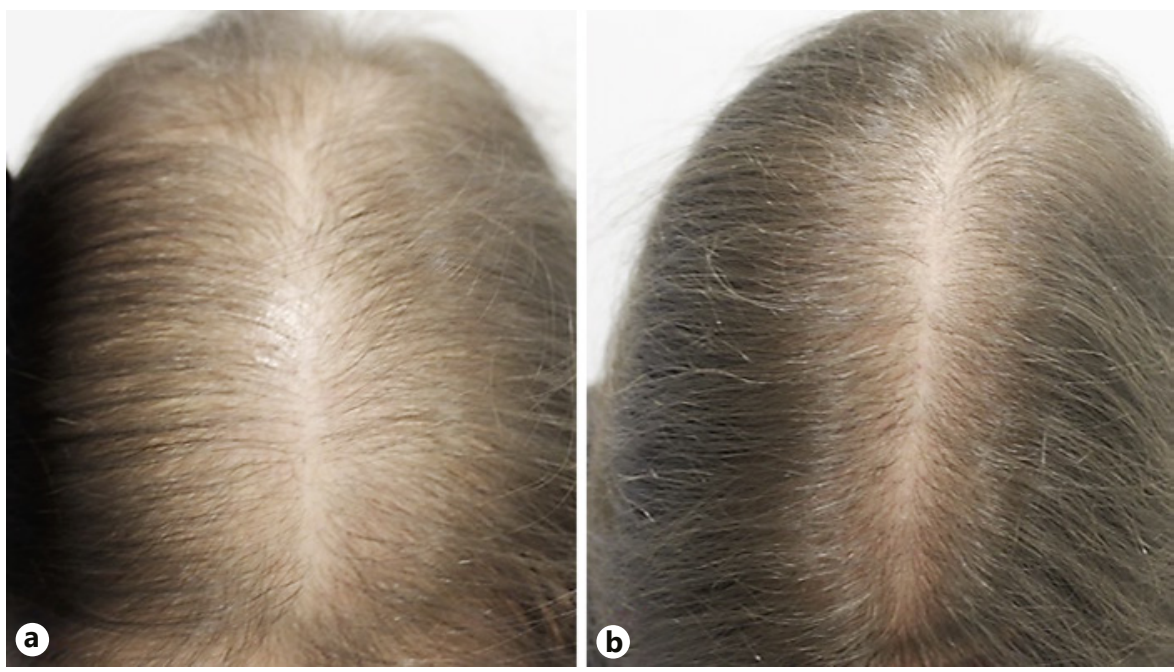

noting that in order to properly evaluate the potential effectiveness of the new intervention with LDOM, only patients with a stabilized alopecia without treatment or clinical changes in the last 12 months were included. Besides, the rate of improvement of patients receiving LDOM in monotherapy was almost as high as patients receiving combined therapies (65 vs. $79 \%$, respectively).

In conclusion, LDOM may be an effective therapy with a good safety profile for women with FPHL, allowing an excellent longterm compliance.

\section{Statement of Ethics}

This study was conducted ethically in accordance with the World Medical Association Declaration of Helsinki, and the study protocol was approved by the Ramón and Cajal Hospital' ethical committee on human research.

\section{Disclosure Statement}

The authors have no conflicts of interest to declare.

\section{Funding Sources}

No funding was received for this study.

\section{Author Contributions}

All authors have contributed in gathering the data for the study, have carefully read the manuscript, and fully approve the final version submitted.

\section{References}

1 Gan DC, Sinclair RD. Prevalence of male and female pattern hair loss in Maryborough. J Investig Dermatol Symp Proc. 2005 Dec;10(3):184-9.

2 Davis DS, Callender VD. Review of quality of life studies in women with alopecia. Int J Womens Dermatol. 2018 Jan;4(1):18-22.

3 Dinh QQ, Sinclair R. Female pattern hair loss: current treatment concepts. Clin Interv Aging. 2007;2(2):189-99.

4 Sinclair RD. Female pattern hair loss: a pilot study investigating combination therapy with low-dose oral minoxidil and spironolactone. Int $J$ Dermatol. 2018 Jan;57(1):104-9.

5 Beach RA. Case series of oral minoxidil for androgenetic and traction alopecia: Tolerability \& the five C's of oral therapy. Dermatol Ther (Heidelb). 2018 Nov;31(6):e12707.

6 Ramos PM, Sinclair RD, Kasprzak M, Miot HA. Minoxidil $1 \mathrm{mg}$ oral versus minoxidil $5 \%$ topical solution for the treatment of female pattern hair loss: A randomized clinical trial. J Am Acad Dermatol. 2020 Jan; $82(1): 252-3$. 